\title{
New inequalities for weaving frames in Hilbert spaces
}

\author{
Yuxiang $\mathrm{Xu}^{1}$ and Jinsong Leng ${ }^{1 *}$ (B)
}

"Correspondence:

jinsongleng@126.com

${ }^{1}$ School of Mathematical Sciences, University of Electronic Science and

Technology of China, Chengdu, 611731, P.R. China

\begin{abstract}
In this paper, we establish Parseval identities and surprising new inequalities for weaving frames in Hilbert spaces which involve scalar $\lambda \in \mathcal{R}$. With suitable choices of $\lambda$, one obtains the previous results as special cases. Our results generalize and improve the remarkable results obtained by Balan et al. and Găvruţa.
\end{abstract}

MSC: 42C15; 47B99

Keywords: Weaving frames; Frames; Hilbert spaces

\section{Introduction}

Frames in Hilbert spaces were first introduced in 1952 by Duffin and Schaeffer [8] to study several deep problems in non-harmonic Fourier series and reintroduced in 1986 by Daubechies, Grossmann, and Meyer [6]. Now, frames have been widely used in coding theory [16], sampling theory [19, 22], quantum field theory [9], filter bank theory [13], image processing [7], and other areas of mathematics, physics, and engineering.

Let $\mathcal{H}$ be a separable space and $I$ be a countable index set. A sequence $\left\{\phi_{i}\right\}_{i \in I}$ of elements of $\mathcal{H}$ is a frame for $\mathcal{H}$ if there exist constants $A, B>0$ such that

$$
A\|f\|^{2} \leq \sum_{i \in I}\left|\left\langle f, \phi_{i}\right\rangle\right|^{2} \leq B\|f\|^{2}, \quad \forall f \in \mathcal{H}
$$

The numbers $A, B$ are called lower and upper frame bounds, respectively. If $A=B$, then this frame is called an $A$-tight frame, and if $A=B=1$, then it is called a Parseval frame.

Suppose that $\left\{\phi_{i}\right\}_{i \in I}$ is a frame for $\mathcal{H}$, then the frame operator is a self-adjoint positive invertible operator, which is given by

$$
S: \mathcal{H} \rightarrow \mathcal{H}, \quad S f=\sum_{i \in I}\left\langle f, \phi_{i}\right\rangle \phi_{i}
$$

The following reconstruction formula holds:

$$
f=\sum_{i \in I}\left\langle f, \phi_{i}\right\rangle S^{-1} \phi_{i}=\sum_{i \in I}\left\langle f, S^{-1} \phi_{i}\right\rangle \phi_{i}
$$

(c) The Author(s) 2021. This article is licensed under a Creative Commons Attribution 4.0 International License, which permits use, sharing, adaptation, distribution and reproduction in any medium or format, as long as you give appropriate credit to the original author(s) and the source, provide a link to the Creative Commons licence, and indicate if changes were made. The images or other third party material in this article are included in the article's Creative Commons licence, unless indicated otherwise in a credit line to the material. If material is not included in the article's Creative Commons licence and your intended use is not permitted by statutory regulation or exceeds the permitted use, you will need to obtain permission directly from the copyright holder. To view a copy of this licence, visit http://creativecommons.org/licenses/by/4.0/. 
where the family $\left\{\widetilde{\phi}_{i}\right\}_{i \in I}=\left\{S^{-1} \phi_{i}\right\}_{i \in I}$ is also a frame for $\mathcal{H}$, which is called the canonical dual frame of $\left\{\phi_{i}\right\}_{i \in I}$. The frame $\left\{\varphi_{i}\right\}_{i \in I}$ for $\mathcal{H}$ is called an alternate dual frame of $\left\{\phi_{i}\right\}_{i \in I}$ if the following formula holds:

$$
f=\sum_{i \in I}\left\langle f, \phi_{i}\right\rangle \varphi_{i}=\sum_{i \in I}\left\langle f, \varphi_{i}\right\rangle \phi_{i}
$$

for all $f \in \mathcal{H}[11]$.

Let $\left\{\phi_{i}\right\}_{i \in I}$ be a frame for $\mathcal{H}$, for every $J \subset I$, we define the operator

$$
S_{J}=\sum_{i \in J}\left\langle f, \phi_{i}\right\rangle \phi_{i}
$$

and denote $J^{c}=I \backslash J$.

First introduced by Bemrose and Casazza et al. [4], the concept of discrete weaving frames for separable Hilbert space is motivated by distributed signal processing, e.g., wireless sensor networks in which frames may be subjected to distributed processing under different frames. Thus, weaving frames have potential applications in wireless sensor networks that require distributed processing under different frames, as well as pre-processing of signals using Gabor frames. Recently, weaving frames in Hilbert spaces have been studied intensively; for more details, see [5, 12, 20].

Definition 1 Two frames $\left\{\phi_{i}\right\}_{i \in I}$ and $\left\{\psi_{i}\right\}_{i \in I}$ for a Hilbert space $\mathcal{H}$ are said to be woven if there are universal constants $A$ and $B$ so that for every partition $\sigma \subset I$ the family $\left\{\phi_{i}\right\}_{i \in \sigma} \cup$ $\left\{\psi_{i}\right\}_{i \in \sigma^{c}}$ is a frame for $\mathcal{H}$ with lower and upper frame bounds $A$ and $B$, respectively. The family $\left\{\phi_{i}\right\}_{i \in \sigma} \cup\left\{\psi_{i}\right\}_{i \in \sigma^{c}}$ is called a weaving.

If $A=B$, we say that $\left\{\phi_{i}\right\}_{i \in I}$ and $\left\{\psi_{i}\right\}_{i \in I}$ are $A$-woven, and if $A=B=1$, then we call them 1 -woven.

Suppose that $\left\{\phi_{i}\right\}_{i \in I}$ and $\left\{\psi_{i}\right\}_{i \in I}$ are woven, the frame operator of $\left\{\phi_{i}\right\}_{i \in \sigma} \cup\left\{\psi_{i}\right\}_{i \in \sigma^{c}}$ is defined by

$$
S_{\mathcal{W}} f=\sum_{i \in \sigma}\left\langle f, \phi_{i}\right\rangle \phi_{i}+\sum_{i \in \sigma^{c}}\left\langle f, \psi_{i}\right\rangle \psi_{i}
$$

and then $S_{\mathcal{W}}$ is a bounded, invertible, self-adjoint positive operator. A frame $\left\{\varphi_{i}\right\}_{i \in I}$ is called an alternate dual frame of $\left\{\phi_{i}\right\}_{i \in \sigma} \cup\left\{\psi_{i}\right\}_{i \in \sigma^{c}}$ if for all $f \in \mathcal{H}$ the following identity holds:

$$
f=\sum_{i \in \sigma}\left\langle f, \phi_{i}\right\rangle \varphi_{i}+\sum_{i \in \sigma^{c}}\left\langle f, \psi_{i}\right\rangle \varphi_{i}
$$

For every $\sigma \subset I$, define the bounded linear operators $S_{\mathcal{W}}^{\sigma}, S_{\mathcal{W}}^{\sigma^{c}}: \mathcal{H} \rightarrow \mathcal{H}$ by

$$
S_{\mathcal{W}}^{\sigma} f=\sum_{i \in \sigma}\left\langle f, \phi_{i}\right\rangle \phi_{i}, \quad S_{\mathcal{W}}^{\sigma^{c}} f=\sum_{i \in \sigma^{c}}\left\langle f, \psi_{i}\right\rangle \psi_{i} .
$$

It is easy to verify that $S_{\mathcal{W}}^{\sigma}$ and $S_{\mathcal{W}}^{\sigma^{c}}$ are self-adjoint.

In [1], the authors solved a long-standing conjecture of the signal processing community. They showed that for suitable frames $\left\{\phi_{i}\right\}_{i \in I}$ a signal $f$ can (up to a global phase) be 
recovered from the phase-less measurements $\left\{\left|\left\langle f, \phi_{i}\right\rangle\right|\right\}_{i \in I}$. Note that this only shows that reconstruction of $f$ is in principle possible, but there is no effective constructive algorithm. While searching for such an algorithm, the authors of [2] discovered a new identity for Parseval frames [3]. The authors in [10,23] generalized these identities to alternate dual frames and obtained some general results. The study of inequalities has interested many mathematicians. Some authors have extended the equalities and inequalities for frames in Hilbert spaces to generalized frames [15, 17, 18, 21]. The following form was given in [3] (see [2] for a discussion of the origins of this fundamental identity).

Theorem 1 Let $\left\{\phi_{i}\right\}_{i \in I}$ be a Parseval frame for $\mathcal{H}$. For every $J \subset I$ and every $f \in \mathcal{H}$, we have

$$
\sum_{i \in J}\left|\left\langle f, \phi_{i}\right\rangle\right|^{2}+\left\|\sum_{i \in J^{c}}\left\langle f, \phi_{i}\right\rangle \phi_{i}\right\|^{2}=\sum_{i \in J^{c}}\left|\left\langle f, \phi_{i}\right\rangle\right|^{2}+\left\|\sum_{i \in J}\left\langle f, \phi_{i}\right\rangle \phi_{i}\right\|^{2} \geq \frac{3}{4}\|f\|^{2} .
$$

Later, the author of [10] generalized Theorem 1 to general frames.

Theorem 2 Let $\left\{\phi_{i}\right\}_{i \in I}$ be a frame for $\mathcal{H}$ with a canonical dual frame $\left\{\widetilde{\phi}_{i}\right\}_{i \in I}$; then, for every $J \subset I$ and every $f \in \mathcal{H}$, we have

$$
\sum_{i \in J}\left|\left\langle f, \phi_{i}\right\rangle\right|^{2}+\sum_{i \in I}\left|\left\langle S_{J c} f, \widetilde{\phi}_{i}\right\rangle\right|^{2}=\sum_{i \in J^{c}}\left|\left\langle f, \phi_{i}\right\rangle\right|^{2}+\sum_{i \in I}\left|\left\langle S_{J} f, \widetilde{\phi}_{i}\right\rangle\right|^{2} \geq \frac{3}{4} \sum_{i \in I}\left|\left\langle f, \phi_{i}\right\rangle\right|^{2} .
$$

Theorem 3 Let $\left\{\phi_{i}\right\}_{i \in I}$ be a frame for $\mathcal{H}$ and $\left\{\varphi_{i}\right\}_{i \in I}$ be an alternate dual frame of $\left\{\phi_{i}\right\}_{i \in I}$; then, for every $J \subset I$ and every $f \in \mathcal{H}$, we have

$$
\begin{aligned}
\operatorname{Re}\left(\sum_{i \in J}\left\langle f, \varphi_{i}\right\rangle \overline{\left\langle f, \phi_{i}\right\rangle}\right)+\left\|\sum_{i \in J^{c}}\left\langle f, \varphi_{i}\right\rangle \phi_{i}\right\|^{2} & =\operatorname{Re}\left(\sum_{i \in J^{c}}\left\langle f, \varphi_{i}\right\rangle \overline{\left\langle f, \phi_{i}\right\rangle}\right)+\left\|\sum_{i \in J}\left\langle f, \varphi_{i}\right\rangle \phi_{i}\right\|^{2} \\
& \geq \frac{3}{4}\|f\|^{2} .
\end{aligned}
$$

Motivated by these interesting results, the authors of [23] generalized Theorem 3 to a more general form that does not involve the real parts of the complex numbers.

Theorem 4 Let $\left\{\phi_{i}\right\}_{i \in I}$ be a frame for $\mathcal{H}$ and $\left\{\varphi_{i}\right\}_{i \in I}$ be an alternate dual frame of $\left\{\phi_{i}\right\}_{i \in I}$; then, for every $J \subset I$ and every $f \in \mathcal{H}$, we have

$$
\begin{aligned}
\left(\sum_{i \in J}\left\langle f, \varphi_{i}\right\rangle \overline{\left\langle f, \phi_{i}\right\rangle}\right)+\left\|\sum_{i \in J^{c}}\left\langle f, \varphi_{i}\right\rangle \phi_{i}\right\|^{2} & =\overline{\left(\sum_{i \in J^{c}}\left\langle f, \varphi_{i}\right\rangle \overline{\left\langle f, \phi_{i}\right\rangle}\right)}+\left\|\sum_{i \in J}\left\langle f, \varphi_{i}\right\rangle \phi_{i}\right\|^{2} \\
& \geq \frac{3}{4}\|f\|^{2} .
\end{aligned}
$$

In this paper, we generalize the results of the above-mentioned weaving frames in Hilbert spaces. Then, inspired by [18], we also generalize the previous inequalities from the scalar $\lambda \in[0,1]$ to a more general form that involves a scalar $\lambda \in \mathcal{R}$. Since a frame is woven with itself, we can consider that the above-mentioned equality and inequalities in frames can be obtained as a special case of the results that we establish on weaving frames. 


\section{Results and their proofs}

We first state a simple result on operators, which is a distortion of [23, Lemma 2.1].

Lemma 1 If $P, Q \in L(\mathcal{H})$ satisfies $P+Q=I_{\mathcal{H}}$, then $P+Q^{*} Q=Q^{*}+P^{*} P$.

Proof A simple computation shows that

$$
\begin{aligned}
P+Q^{*} Q & =I_{\mathcal{H}}-Q+Q^{*} Q=I_{\mathcal{H}}-\left(I_{\mathcal{H}}-Q^{*}\right) Q=I_{\mathcal{H}}-P^{*}\left(I_{\mathcal{H}}-P\right) \\
& =I_{\mathcal{H}}-P^{*}+P^{*} P=Q^{*}+P^{*} P .
\end{aligned}
$$

Now, we state and prove a Parseval weaving frame identity.

Theorem 5 Suppose that $\left\{\phi_{i}\right\}_{i \in I}$ and $\left\{\psi_{i}\right\}_{i \in I}$ for a Hilbert space $\mathcal{H}$ are 1-woven, then, for all $\sigma \subset I$ and all $f \in \mathcal{H}$, we have

$$
\sum_{i \in \sigma}\left|\left\langle f, \phi_{i}\right\rangle\right|^{2}+\left\|\sum_{i \in \sigma^{c}}\left\langle f, \psi_{i}\right\rangle \psi_{i}\right\|^{2}=\sum_{i \in \sigma^{c}}\left|\left\langle f, \psi_{i}\right\rangle\right|^{2}+\left\|\sum_{i \in \sigma}\left\langle f, \phi_{i}\right\rangle \phi_{i}\right\|^{2} \geq \frac{3}{4}\|f\|^{2} .
$$

Proof Since $\left\{\phi_{i}\right\}_{i \in I}$ and $\left\{\psi_{i}\right\}_{i \in I}$ are 1-woven, the weaving frame $\left\{\phi_{i}\right\}_{i \in \sigma} \cup\left\{\psi_{i}\right\}_{i \in \sigma^{c}}$ is a Parseval frame for $\mathcal{H}$. Then the frame operator of $\left\{\phi_{i}\right\}_{i \in \sigma} \cup\left\{\psi_{i}\right\}_{i \in \sigma^{c}}$ is $S_{\mathcal{W}}=I_{\mathcal{H}}$. For every $\sigma \subset I$, we have $S_{\mathcal{W}}^{\sigma}+S_{\mathcal{W}}^{\sigma^{c}}=I_{\mathcal{H}}$. Note that $S_{\mathcal{W}}^{\sigma^{c}}$ is a self-adjoint operator, and therefore $\left(S_{\mathcal{W}}^{\sigma^{c}}\right)^{*}=S_{\mathcal{W}}^{\sigma^{c}}$. By applying Lemma 1 to the operators $S_{\mathcal{W}}^{\sigma}$ and $S_{\mathcal{W}}^{\sigma^{c}}$, for all $f \in \mathcal{H}$, we obtain

$$
\left\langle S_{\mathcal{W}}^{\sigma} f, f\right\rangle+\left\langle\left(S_{\mathcal{W}}^{\sigma^{c}}\right)^{*} S_{\mathcal{W}}^{\sigma^{c}} f, f\right\rangle=\left\langle\left(S_{\mathcal{W}}^{\sigma^{c}}\right)^{*} f, f\right\rangle+\left\langle\left(S_{\mathcal{W}}^{\sigma}\right)^{*} S_{\mathcal{W}}^{\sigma} f, f\right\rangle .
$$

Thus,

$$
\left\langle S_{\mathcal{W}}^{\sigma} f, f\right\rangle+\left\|S_{\mathcal{W}}^{\sigma^{c}} f\right\|=\left\langle S_{\mathcal{W}}^{\sigma^{c}} f, f\right\rangle+\left\|S_{\mathcal{W}}^{\sigma} f\right\|
$$

Hence,

$$
\sum_{i \in \sigma}\left|\left\langle f, \phi_{i}\right\rangle\right|^{2}+\left\|\sum_{i \in \sigma^{c}}\left\langle f, \psi_{i}\right\rangle \psi_{i}\right\|^{2}=\sum_{i \in \sigma^{c}}\left|\left\langle f, \psi_{i}\right\rangle\right|^{2}+\left\|\sum_{i \in \sigma}\left\langle f, \phi_{i}\right\rangle \phi_{i}\right\|^{2} .
$$

Next, we prove the inequality of (2.1). A simple computation shows that

$$
\begin{aligned}
\left(S_{\mathcal{W}}^{\sigma}\right)^{2}+\left(S_{\mathcal{W}}^{\sigma^{c}}\right)^{2} & =\left(S_{\mathcal{W}}^{\sigma}\right)^{2}+\left(I_{\mathcal{H}}-\left(S_{\mathcal{W}}^{\sigma}\right)\right)^{2}=2\left(S_{\mathcal{W}}^{\sigma}\right)^{2}-2 S_{\mathcal{W}}^{\sigma}+I_{\mathcal{H}} \\
& =2\left(S_{\mathcal{W}}^{\sigma}-\frac{1}{2} I_{\mathcal{H}}\right)^{2}+\frac{1}{2} I_{\mathcal{H}},
\end{aligned}
$$

and so

$$
\left(S_{\mathcal{W}}^{\sigma}\right)^{2}+\left(S_{\mathcal{W}}^{\sigma^{c}}\right)^{2} \geq \frac{1}{2} I_{\mathcal{H}}
$$

Since $S_{\mathcal{W}}^{\sigma}+S_{\mathcal{W}}^{\sigma^{c}}=I_{\mathcal{H}}$, it follows that

$$
S_{\mathcal{W}}^{\sigma}+\left(S_{\mathcal{W}}^{\sigma^{c}}\right)^{2}+S_{\mathcal{W}}^{\sigma^{c}}+\left(S_{\mathcal{W}}^{\sigma}\right)^{2} \geq \frac{3}{2} I_{\mathcal{H}}
$$


Note that the operator $S_{\mathcal{W}}^{\sigma}$ is also self-adjoint, and therefore $\left(S_{\mathcal{W}}^{\sigma}\right)^{*}=S_{\mathcal{W}}^{\sigma}$. Applying Lemma 1 to the operators $P=S_{\mathcal{W}}^{\sigma}$ and $Q=S_{\mathcal{W}}^{\sigma^{c}}$, we obtain

$$
S_{\mathcal{W}}^{\sigma}+\left(S_{\mathcal{W}}^{\sigma^{c}}\right)^{2}=S_{\mathcal{W}}^{\sigma^{c}}+\left(S_{\mathcal{W}}^{\sigma}\right)^{2}
$$

Then, equation (2.2) means that

$$
\left(S_{\mathcal{W}}^{\sigma^{c}}+\left(S_{\mathcal{W}}^{\sigma}\right)^{2}\right) \geq \frac{3}{4} I_{\mathcal{H}}
$$

Therefore, for all $f \in \mathcal{H}$, we have

$$
\sum_{i \in \sigma^{c}}\left|\left\langle f, \psi_{i}\right\rangle\right|^{2}+\left\|\sum_{i \in \sigma}\left\langle f, \phi_{i}\right\rangle \phi_{i}\right\|^{2}=\left\langle S_{\mathcal{W}}^{\sigma^{c}} f, f\right\rangle+\left\langle S_{\mathcal{W}}^{\sigma} f, S_{\mathcal{W}}^{\sigma} f\right\rangle=\left\langle\left(S_{\mathcal{W}}^{\sigma^{c}}+\left(S_{\mathcal{W}}^{\sigma}\right)^{2}\right) f, f\right\rangle \geq \frac{3}{4}\|f\|^{2} .
$$

This completes the proof.

Remark 6 If we take $\phi_{i}=\psi_{i}$ for all $i \in I$ in Theorem 5, we can obtain Theorem 1.

Lemma 2 Let $P, Q \in L(\mathcal{H})$ be two self-adjoint operators such that $P+Q=I_{\mathcal{H}}$, then, for any $\lambda \in \mathcal{R}$ and all $f \in \mathcal{H}$, we have

$$
\begin{aligned}
\|P f\|^{2}+\lambda\langle Q f, f\rangle & =\|Q f\|^{2}+(2-\lambda)\langle P f, f\rangle+(\lambda-1)\|f\|^{2} \\
& \geq\left(\lambda-\frac{\lambda^{2}}{4}\right)\|f\|^{2} .
\end{aligned}
$$

Proof For all $f \in \mathcal{H}$, we have

$$
\begin{aligned}
\|P f\|^{2}+\lambda\langle Q f, f\rangle & =\left\langle P^{2} f, f\right\rangle+\lambda\left\langle\left(I_{\mathcal{H}}-P\right) f, f\right\rangle \\
& =\left\langle\left(P^{2}-\lambda P+\lambda I_{\mathcal{H}}\right) f, f\right\rangle \\
& =\left\langle\left(I_{\mathcal{H}}-P\right)^{2} f, f\right\rangle+(2-\lambda)\langle P f, f\rangle+(\lambda-1)\langle f, f\rangle \\
& =\|Q f\|^{2}+(2-\lambda)\langle P f, f\rangle+(\lambda-1)\|f\|^{2} .
\end{aligned}
$$

A simple computation of (2.3) gives

$$
\begin{aligned}
\left\langle\left(P^{2}-\lambda P+\lambda I_{\mathcal{H}}\right) f, f\right\rangle & =\left\langle\left(\left(P-\frac{\lambda}{2} I_{\mathcal{H}}\right)^{2}-\frac{\lambda^{2}}{4} I_{\mathcal{H}}+\lambda I_{\mathcal{H}}\right) f, f\right\rangle \\
& =\left\langle\left(\left(P-\lambda I_{\mathcal{H}}\right)^{2}+\left(\lambda-\frac{\lambda^{2}}{4}\right) I_{\mathcal{H}}\right) f, f\right\rangle \\
& \geq\left(\lambda-\frac{\lambda^{2}}{4}\right)\|f\|^{2} .
\end{aligned}
$$

This proves the desired result.

Theorem 7 Suppose that two frames $\left\{\phi_{i}\right\}_{i \in I}$ and $\left\{\psi_{i}\right\}_{i \in I}$ for a Hilbert space $\mathcal{H}$ are woven; then, for any $\lambda \in \mathcal{R}$, for all $\sigma \subset I$ and all $f \in \mathcal{H}$, we have

$$
\sum_{i \in \sigma}\left|\left\langle f, \phi_{i}\right\rangle\right|^{2}+\sum_{i \in \sigma}\left|\left\langle S_{\mathcal{W}}^{\sigma^{c}} f, S_{\mathcal{W}}^{-1} \phi_{i}\right\rangle\right|^{2}+\sum_{i \in \sigma^{c}}\left|\left\langle S_{\mathcal{W}}^{\sigma^{c}} f, S_{\mathcal{W}}^{-1} \psi_{i}\right\rangle\right|^{2}
$$




$$
\begin{aligned}
& =\sum_{i \in \sigma^{c}}\left|\left\langle f, \psi_{i}\right\rangle\right|^{2}+\sum_{i \in \sigma}\left|\left\langle S_{\mathcal{W}}^{\sigma} f, S_{\mathcal{W}}^{-1} \phi_{i}\right\rangle\right|^{2}+\sum_{i \in \sigma^{c}}\left|\left\langle S_{\mathcal{W}}^{\sigma} f, S_{\mathcal{W}}^{-1} \psi_{i}\right\rangle\right|^{2} \\
& \geq\left(\lambda-\frac{\lambda^{2}}{4}\right) \sum_{i \in \sigma}\left|\left\langle f, \phi_{i}\right\rangle\right|^{2}+\left(1-\frac{\lambda^{2}}{4}\right) \sum_{i \in \sigma^{c}}\left|\left\langle f, \psi_{i}\right\rangle\right|^{2} .
\end{aligned}
$$

Proof Since $\left\{\phi_{i}\right\}_{i \in I}$ and $\left\{\psi_{i}\right\}_{i \in I}$ are woven, for all $\sigma \subset I,\left\{\phi_{i}\right\}_{i \in \sigma} \cup\left\{\psi_{i}\right\}_{i \in \sigma^{c}}$ is a frame for $\mathcal{H}$. Letting $S_{\mathcal{W}}$ be the frame operator for $\left\{\phi_{i}\right\}_{i \in \sigma} \cup\left\{\psi_{i}\right\}_{i \in \sigma}$, since $S_{\mathcal{W}}^{\sigma}+S_{\mathcal{W}}^{\sigma^{c}}=S_{\mathcal{W}}$, it follows that

$$
S_{\mathcal{W}}^{-1 / 2} S_{\mathcal{W}}^{\sigma} S_{\mathcal{W}}^{-1 / 2}+S_{\mathcal{W}}^{-1 / 2} S_{\mathcal{W}}^{\sigma^{c}} S_{\mathcal{W}}^{-1 / 2}=I_{\mathcal{H}}
$$

Considering $P=S_{\mathcal{W}}^{-1 / 2} S_{\mathcal{W}}^{\sigma} S_{\mathcal{W}}^{-1 / 2}, Q=S_{\mathcal{W}}^{-1 / 2} S_{\mathcal{W}}^{\sigma^{c}} S_{\mathcal{W}}^{-1 / 2}$, and $S_{\mathcal{W}}^{1 / 2} f$ instead of $f$ in Lemma 2, we obtain

$$
\begin{aligned}
& \left\|S_{\mathcal{W}}^{-1 / 2} S_{\mathcal{W}}^{\sigma} f\right\|^{2}+\lambda\left\langle S_{\mathcal{W}}^{-1 / 2} S_{\mathcal{W}}^{\sigma^{c}} f, S_{\mathcal{W}}^{1 / 2} f\right\rangle \\
& \quad=\left\|S_{\mathcal{W}}^{-1 / 2} S_{\mathcal{W}}^{\sigma^{c}} f\right\|^{2}+(2-\lambda)\left\langle S_{\mathcal{W}}^{-1 / 2} S_{\mathcal{W}}^{\sigma} f, S_{\mathcal{W}}^{1 / 2} f\right\rangle+(\lambda-1)\left\|S_{\mathcal{W}}^{1 / 2} f\right\|^{2} \\
& \quad \geq\left(\lambda-\frac{\lambda^{2}}{4}\right)\left\|S_{\mathcal{W}}^{1 / 2} f\right\|^{2},
\end{aligned}
$$

and thus

$$
\begin{aligned}
& \left\langle S_{\mathcal{W}}^{-1} S_{\mathcal{W}}^{\sigma} f, S_{\mathcal{W}}^{\sigma} f\right\rangle+\lambda\left\langle S_{\mathcal{W}}^{\sigma^{c}} f, f\right\rangle \\
& \quad=\left\langle S_{\mathcal{W}}^{-1} S_{\mathcal{W}}^{\sigma^{c}} f, S_{\mathcal{W}}^{\sigma^{c}} f\right\rangle+(2-\lambda)\left\langle S_{\mathcal{W}}^{\sigma} f, f\right\rangle+(\lambda-1)\left\langle S_{\mathcal{W}} f, f\right\rangle \\
& \quad \geq\left(\lambda-\frac{\lambda^{2}}{4}\right)\left\langle S_{\mathcal{W}} f, f\right\rangle .
\end{aligned}
$$

Then,

$$
\begin{aligned}
& \left\langle S_{\mathcal{W}}^{-1} S_{\mathcal{W}}^{\sigma} f, S_{\mathcal{W}}^{\sigma} f\right\rangle \\
& \quad=\left\langle S_{\mathcal{W}}^{-1} S_{\mathcal{W}}^{\sigma^{c}} f, S_{\mathcal{W}}^{\sigma^{c}} f\right\rangle+2\left\langle S_{\mathcal{W}}^{\sigma} f, f\right\rangle-\lambda\left\langle\left(S_{\mathcal{W}}^{\sigma}+S_{\mathcal{W}}^{\sigma^{c}}\right) f, f\right\rangle+(\lambda-1)\left\langle S_{\mathcal{W}} f, f\right\rangle \\
& \quad \geq\left(\lambda-\frac{\lambda^{2}}{4}\right)\left\langle S_{\mathcal{W}} f, f\right\rangle-\lambda\left\langle S_{\mathcal{W}}^{\left.\sigma_{\mathcal{W}}^{c} f, f\right\rangle},\right.
\end{aligned}
$$

and thus

$$
\begin{aligned}
& \left\langle S_{\mathcal{W}}^{-1} S_{\mathcal{W}}^{\sigma} f, S_{\mathcal{W}}^{\sigma} f\right\rangle=\left\langle S_{\mathcal{W}}^{-1} S_{\mathcal{W}}^{\left.\sigma^{c} f, S_{\mathcal{W}}^{\sigma} f\right\rangle+2\left\langle S_{\mathcal{W}}^{\sigma} f, f\right\rangle-\left\langle S_{\mathcal{W}} f, f\right\rangle}\right. \\
& \geq \lambda\left\langle S_{\mathcal{W}}^{\sigma} f, f\right\rangle-\frac{\lambda^{2}}{4}\left\langle S_{\mathcal{W}} f, f\right\rangle
\end{aligned}
$$

Hence,

$$
\begin{aligned}
\left\langle S_{\mathcal{W}}^{-1} S_{\mathcal{W}}^{\sigma} f, S_{\mathcal{W}}^{\sigma} f\right\rangle+\left\langle S_{\mathcal{W}}^{\sigma^{c}} f, f\right\rangle & =\left\langle S_{\mathcal{W}}^{-1} S_{\mathcal{W}}^{\sigma^{c}} f, S_{\mathcal{W}}^{\sigma^{c}} f\right\rangle+\left\langle S_{\mathcal{W}}^{\sigma} f, f\right\rangle \\
& \geq\left(\lambda-\frac{\lambda^{2}}{4}\right)\left\langle S_{\mathcal{W}}^{\sigma} f, f\right\rangle+\left(1-\frac{\lambda^{2}}{4}\right)\left\langle S_{\mathcal{W}}^{\sigma^{c}} f, f\right\rangle .
\end{aligned}
$$


We have

$$
\begin{aligned}
\left\langle S_{\mathcal{W}}^{-1} S_{\mathcal{W}}^{\sigma} f, S_{\mathcal{W}}^{\sigma} f\right\rangle & =\left\langle S_{\mathcal{W}} S_{\mathcal{W}}^{-1} S_{\mathcal{W}}^{\sigma} f, S_{\mathcal{W}}^{-1} S_{\mathcal{W}}^{\sigma} f\right\rangle \\
& =\left\langle\sum_{i \in \sigma}\left\langle S_{\mathcal{W}}^{-1} S_{\mathcal{W}}^{\sigma} f, \phi_{i}\right\rangle \phi_{i}+\sum_{i \in \sigma^{c}}\left\langle S_{\mathcal{W}}^{-1} S_{\mathcal{W}}^{\sigma} f, \psi_{i}\right\rangle \psi_{i}, S_{\mathcal{W}}^{-1} S_{\mathcal{W}}^{\sigma} f\right\rangle \\
& =\left\langle\sum_{i \in \sigma}\left\langle S_{\mathcal{W}}^{-1} S_{\mathcal{W} f}^{\sigma} f, \phi_{i}\right\rangle \phi_{i}, S_{\mathcal{W}}^{-1} S_{\mathcal{W}}^{\sigma} f\right\rangle+\left\langle\sum_{i \in \sigma}\left\langle S_{\mathcal{W}}^{-1} S_{\mathcal{W}}^{\sigma} f, \psi_{i}\right\rangle \psi_{i}, S_{\mathcal{W}}^{-1} S_{\mathcal{W}}^{\sigma} f\right\rangle \\
& =\sum_{i \in \sigma}\left|\left\langle S_{\mathcal{W}}^{\sigma} f, S_{\mathcal{W}}^{-1} \phi_{i}\right\rangle\right|^{2}+\sum_{i \in \sigma^{c}}\left|\left\langle S_{\mathcal{W}}^{\sigma} f, S_{\mathcal{W}}^{-1} \psi_{i}\right\rangle\right|^{2} .
\end{aligned}
$$

Similarly,

$$
\begin{aligned}
& \left\langle S_{\mathcal{W}}^{-1} S_{\mathcal{W}}^{\sigma^{c}} f, S_{\mathcal{W}}^{\sigma^{c}} f\right\rangle=\sum_{i \in \sigma}\left|\left\langle S_{\mathcal{W}}^{\sigma^{c}} f, S_{\mathcal{W}}^{-1} \phi_{i}\right\rangle\right|^{2}+\sum_{i \in \sigma^{c}}\left|\left\langle S_{\mathcal{W}}^{\sigma^{c}} f, S_{\mathcal{W}}^{-1} \psi_{i}\right\rangle\right|^{2}, \\
& \left\langle S_{\mathcal{W}}^{\sigma^{c}} f, f\right\rangle=\sum_{i \in \sigma^{c}}\left|\left\langle f, \psi_{i}\right\rangle\right|^{2}, \\
& \left\langle S_{\mathcal{W}}^{\sigma} f, f\right\rangle=\sum_{i \in \sigma^{c}}\left|\left\langle f, \phi_{i}\right\rangle\right|^{2} .
\end{aligned}
$$

Using Eqs. (2.5)-(2.9) in inequality (2.3), we obtain

$$
\begin{aligned}
& \sum_{i \in \sigma}\left|\left\langle f, \phi_{i}\right\rangle\right|^{2}+\sum_{i \in \sigma}\left|\left\langle S_{\mathcal{W}}^{\sigma^{c}} f, S_{\mathcal{W}}^{-1} \phi_{i}\right\rangle\right|^{2}+\sum_{i \in \sigma^{c}}\left|\left\langle S_{\mathcal{W}}^{\sigma^{c}} f, S_{\mathcal{W}}^{-1} \psi_{i}\right\rangle\right|^{2} \\
& =\sum_{i \in \sigma^{c}}\left|\left\langle f, \psi_{i}\right\rangle\right|^{2}+\sum_{i \in \sigma}\left|\left\langle S_{\mathcal{W}}^{\sigma} f, S_{\mathcal{W}}^{-1} \phi_{i}\right\rangle\right|^{2}+\sum_{i \in \sigma^{c}}\left|\left\langle S_{\mathcal{W}}^{\sigma} f, S_{\mathcal{W}}^{-1} \psi_{i}\right\rangle\right|^{2} \\
& \geq\left(\lambda-\frac{\lambda^{2}}{4}\right) \sum_{i \in \sigma}\left|\left\langle f, \phi_{i}\right\rangle\right|^{2}+\left(1-\frac{\lambda^{2}}{4}\right) \sum_{i \in \sigma^{c}}\left|\left\langle f, \psi_{i}\right\rangle\right|^{2}
\end{aligned}
$$

Remark 8 If we take $\phi_{i}=\psi_{i}$ for all $i \in I$ and $\lambda=1$ in Theorem 7, we can obtain Theorem 2 with scalar $3 / 4$.

Lemma 3 If $P, Q \in L(\mathcal{H})$ satisfy $P+Q=I_{\mathcal{H}}$, then, for any $\lambda \in \mathcal{R}$, we have

$$
P^{*} P+\lambda\left(Q^{*}+Q\right)=Q^{*} Q+(1-\lambda)\left(P^{*}+P\right)+(2 \lambda-1) I_{\mathcal{H}} \geq\left(1-(\lambda-1)^{2}\right) I_{\mathcal{H}} .
$$

Proof

$$
P^{*} P+\lambda\left(Q^{*}+Q\right)=P^{*} P+\lambda\left(I_{\mathcal{H}}-P^{*}+I_{P}\right)=P^{*} P-\lambda\left(P^{*}+P\right)+2 \lambda I_{\mathcal{H}},
$$

and

$$
\begin{aligned}
Q^{*} Q+(1-\lambda)\left(P^{*}+P\right)+(2 \lambda-1) I_{\mathcal{H}} \\
\quad=\left(I_{\mathcal{H}}-P^{*}\right)\left(I_{\mathcal{H}}-P\right)+(1-\lambda)\left(P^{*}+P\right)+(2 \lambda-1) I_{\mathcal{H}} \\
\quad=P^{*} P-\lambda\left(P^{*}+P\right)+2 \lambda I_{\mathcal{H}} \\
\quad=\left(P-\lambda I_{\mathcal{H}}\right)^{*}\left(P-\lambda I_{\mathcal{H}}\right)+\left(1-(\lambda-1)^{2}\right) I_{\mathcal{H}}
\end{aligned}
$$




$$
\geq\left(1-(\lambda-1)^{2}\right) I_{\mathcal{H}}
$$

Hence, the result follows.

Theorem 9 Suppose that two frames $\left\{\phi_{i}\right\}_{i \in I}$ and $\left\{\psi_{i}\right\}_{i \in I}$ for a Hilbert space $\mathcal{H}$ are woven and $\left\{\varphi_{i}\right\}_{i \in I}$ is an alternate dual frame of the weaving frame $\left\{\phi_{i}\right\}_{i \in \sigma} \cup\left\{\psi_{i}\right\}_{i \in \sigma}$. Then, for any $\lambda \in \mathcal{R}$, for all $\sigma \subset I$, and for all $f \in \mathcal{H}$, we have

$$
\begin{aligned}
\operatorname{Re} & \left(\sum_{i \in \sigma}\left\langle f, \varphi_{i}\right\rangle \overline{\left\langle f, \phi_{i}\right\rangle}\right)+\left\|\sum_{i \in \sigma^{c}}\left\langle f, \varphi_{i}\right\rangle \psi_{i}\right\|^{2} \\
& =\operatorname{Re}\left(\sum_{i \in \sigma^{c}}\left\langle f, \varphi_{i}\right\rangle \overline{\left\langle f, \psi_{i}\right\rangle}\right)+\left\|\sum_{i \in \sigma}\left\langle f, \varphi_{i}\right\rangle \phi_{i}\right\|^{2} \\
& \geq\left(2 \lambda-\lambda^{2}\right) \operatorname{Re}\left(\sum_{i \in \sigma}\left\langle f, \varphi_{i}\right\rangle \overline{\left\langle f, \phi_{i}\right\rangle}\right)+\left(1-\lambda^{2}\right) \operatorname{Re}\left(\sum_{i \in \sigma^{c}}\left\langle f, \varphi_{i}\right\rangle \overline{\left\langle f, \psi_{i}\right\rangle}\right) .
\end{aligned}
$$

Proof For all $f \in \mathcal{H}$ and all $\sigma \subset I$, we define the operators

$$
E_{\sigma} f=\sum_{i \in \sigma}\left\langle f, \varphi_{i}\right\rangle \phi_{i}, \quad E_{\sigma} f=\sum_{i \in \sigma^{c}}\left\langle f, \varphi_{i}\right\rangle \psi_{i}
$$

Then the series converge unconditionally and $E_{\sigma}, E_{\sigma^{c}} \in L(\mathcal{H})$. By (1.1), we have $E_{\sigma}+E_{\sigma^{c}}=$ $I_{\mathcal{H}}$. Applying Lemma 3 to the operators $P=E_{\sigma}$ and $Q=E_{\sigma^{c}}$, for all $f \in \mathcal{H}$, we obtain

$$
\begin{aligned}
\left\langle E_{\sigma}^{*}\right. & \left.E_{\sigma} f, f\right\rangle+\lambda\left|\left(E_{\sigma^{c}}^{*}+E_{\sigma^{c}}\right) f, f\right\rangle \\
& =\left\langle E_{\sigma}^{*} E_{\sigma} f, f\right\rangle+\lambda \overline{\left\langle E_{\sigma^{c}} f, f\right\rangle}+\lambda\left\langle E_{\sigma} f, f\right\rangle \\
& =\left\langle E_{\sigma^{c}}^{*} E_{\sigma} c f, f\right\rangle+(1-\lambda)\left\langle\left(E_{\sigma}^{*}+E_{\sigma}\right) f, f\right\rangle+(2 \lambda-1)\|f\|^{2} \\
& =\left\langle E_{\sigma^{c}}^{*} E_{\sigma} f f, f\right\rangle+(1-\lambda)\left(\overline{\left\langle E_{\sigma} f, f\right\rangle}+\left\langle E_{\sigma} f, f\right\rangle\right)+(2 \lambda-1)\left\langle I_{\mathcal{H}} f, f\right\rangle .
\end{aligned}
$$

With a simple computation of (2.11) and (2.12), we have

$$
\left\|E_{\sigma} f\right\|^{2}+2 \lambda \operatorname{Re}\left\langle E_{\sigma} c f, f\right\rangle=\left\|E_{\sigma} c\right\|^{2}+2(1-\lambda) \operatorname{Re}\left\langle E_{\sigma} f, f\right\rangle+(2 \lambda-1) \operatorname{Re}\left\langle I_{\mathcal{H}} f, f\right\rangle .
$$

Then

$$
\begin{aligned}
\left\|E_{\sigma} f\right\|^{2} & =\left\|E_{\sigma} f\right\|^{2}+2(1-\lambda) \operatorname{Re}\left\langle E_{\sigma} f, f\right\rangle-2 \lambda \operatorname{Re}\left\langle E_{\sigma} c f, f\right\rangle+(2 \lambda-1) \operatorname{Re}\left\langle I_{\mathcal{H}} f, f\right\rangle \\
& =\left\|E_{\sigma c} f\right\|^{2}+2 \operatorname{Re}\left\langle E_{\sigma} f, f\right\rangle-2 \lambda \operatorname{Re}\left\langle\left(E_{\sigma}+E_{\sigma}\right) f, f\right\rangle+(2 \lambda-1) \operatorname{Re}\left\langle I_{\mathcal{H}} f, f\right\rangle \\
& =\left\|E_{\sigma} f\right\|^{2}+2 \operatorname{Re}\left\langle E_{\sigma} f, f\right\rangle-\operatorname{Re}\left\langle I_{\mathcal{H}} f, f\right\rangle \\
& =\left\|E_{\sigma} f\right\|^{2}+2 \operatorname{Re}\left\langle E_{\sigma} f, f\right\rangle-\operatorname{Re}\left\langle\left(E_{\sigma}+E_{\sigma^{c}}\right) f, f\right\rangle \\
& =\left\|E_{\sigma} f\right\|^{2}+\operatorname{Re}\left\langle E_{\sigma} f, f\right\rangle-\operatorname{Re}\left\langle E_{\sigma} f, f\right\rangle .
\end{aligned}
$$

Hence,

$$
\left\|E_{\sigma} f\right\|^{2}+\operatorname{Re}\left\langle E_{\sigma} c f, f\right\rangle=\left\|E_{\sigma} c f\right\|^{2}+\operatorname{Re}\left\langle E_{\sigma} f, f\right\rangle .
$$


Since

$$
\begin{aligned}
& \left\|E_{\sigma} f\right\|^{2}=\left\|\sum_{i \in \sigma}\left\langle f, \varphi_{i}\right\rangle \phi_{i}\right\|^{2}, \\
& \operatorname{Re}\left\langle E_{\sigma^{c}} f, f\right\rangle=\operatorname{Re}\left(\sum_{i \in \sigma^{c}}\left\langle f, \varphi_{i}\right\rangle \overline{\left\langle f, \psi_{i}\right\rangle}\right), \\
& \left\|E_{\sigma^{c}} f\right\|^{2}=\left\|\sum_{i \in \sigma}\left\langle f, \varphi_{i}\right\rangle \psi_{i}\right\|^{2}, \\
& \operatorname{Re}\left\langle E_{\sigma} f, f\right\rangle=\operatorname{Re}\left(\sum_{i \in \sigma}\left\langle f, \varphi_{i}\right\rangle \overline{\left\langle f, \phi_{i}\right\rangle}\right) .
\end{aligned}
$$

Using equations (2.13)-(2.17), we have

$$
\operatorname{Re}\left(\sum_{i \in \sigma}\left\langle f, \varphi_{i}\right\rangle \overline{\left\langle f, \phi_{i}\right\rangle}\right)+\left\|\sum_{i \in \sigma^{c}}\left\langle f, \varphi_{i}\right\rangle \psi_{i}\right\|^{2}=\operatorname{Re}\left(\sum_{i \in \sigma^{c}}\left\langle f, \varphi_{i}\right\rangle \overline{\left\langle f, \psi_{i}\right\rangle}\right)+\left\|\sum_{i \in \sigma}\left\langle f, \varphi_{i}\right\rangle \phi_{i}\right\|^{2} .
$$

We now prove the inequality of (2.10). From Lemma 3, we have

$$
\left\langle E_{\sigma}^{*} E_{\sigma} f, f\right\rangle+\lambda \overline{\left\langle E_{\sigma} f f, f\right\rangle}+\lambda\left\langle E_{\sigma} f f, f\right\rangle \geq\left(2 \lambda-\lambda^{2}\right)\left\langle I_{\mathcal{H}} f, f\right\rangle .
$$

Then

$$
\left\|E_{\sigma} f\right\|^{2}+2 \lambda \operatorname{Re}\left\langle E_{\sigma} f, f\right\rangle \geq\left(2 \lambda-\lambda^{2}\right) \operatorname{Re}\left\langle I_{\mathcal{H}} f, f\right\rangle,
$$

hence

$$
\begin{aligned}
\left\|E_{\sigma} f\right\|^{2} & \geq\left(2 \lambda-\lambda^{2}\right) \operatorname{Re}\left\langle I_{\mathcal{H}} f, f\right\rangle-2 \lambda \operatorname{Re}\left\langle E_{\sigma^{c}} f, f\right\rangle \\
& =\left(2 \lambda-\lambda^{2}\right) \operatorname{Re}\left\langle\left(E_{\sigma}+E_{\sigma^{c}}\right) f, f\right\rangle-2 \lambda \operatorname{Re}\left\langle E_{\sigma^{c}} f, f\right\rangle \\
& =\left(2 \lambda-\lambda^{2}\right) \operatorname{Re}\left\langle E_{\sigma} f, f\right\rangle-\lambda^{2} \operatorname{Re}\left\langle E_{\sigma^{c}} f, f\right\rangle \\
& =\left(2 \lambda-\lambda^{2}\right) \operatorname{Re}\left\langle E_{\sigma} f, f\right\rangle+\left(1-\lambda^{2}\right) \operatorname{Re}\left\langle E_{\sigma^{c}} f, f\right\rangle-\operatorname{Re}\left\langle E_{\sigma^{c}} f, f\right\rangle .
\end{aligned}
$$

Therefore,

$$
\left\|E_{\sigma} f\right\|^{2}+\operatorname{Re}\left\langle E_{\sigma} f, f\right\rangle \geq\left(2 \lambda-\lambda^{2}\right) \operatorname{Re}\left\langle E_{\sigma} f, f\right\rangle+\left(1-\lambda^{2}\right) \operatorname{Re}\left\langle E_{\sigma^{c}} f, f\right\rangle .
$$

Using Eqs. (2.14) -(2.17) and (2.19), we have

$$
\begin{aligned}
& \operatorname{Re}\left(\sum_{i \in \sigma}\left\langle f, \varphi_{i}\right\rangle \overline{\left\langle f, \phi_{i}\right\rangle}\right)+\left\|\sum_{i \in \sigma^{c}}\left\langle f, \varphi_{i}\right\rangle \psi_{i}\right\|^{2} \\
& \quad \geq\left(2 \lambda-\lambda^{2}\right) \operatorname{Re}\left(\sum_{i \in \sigma}\left\langle f, \varphi_{i}\right\rangle \overline{\left\langle f, \phi_{i}\right\rangle}\right)+\left(1-\lambda^{2}\right) \operatorname{Re}\left(\sum_{i \in \sigma^{c}}\left\langle f, \varphi_{i}\right\rangle \overline{\left\langle f, \psi_{i}\right\rangle}\right) .
\end{aligned}
$$

The proof is completed. 
Remark 10 Theorem 3 can be obtained from Theorem 9 by taking $\phi_{i}=\psi_{i}$ for all $i \in I$ and $\lambda=\frac{1}{2}$.

Theorem 11 Suppose that $\Phi=\left\{\phi_{i}\right\}_{i \in I}$ and $\Psi=\left\{\psi_{i}\right\}_{i \in I}$ for a Hilbert space $\mathcal{H}$ are woven and $\left\{\varphi_{i}\right\}_{i \in I}$ is an alternate dual frame of the weaving frame $\left\{\phi_{i}\right\}_{i \in \sigma} \cup\left\{\phi_{i}\right\}_{i \in \sigma}$. Then, for any $\lambda \in \mathcal{R}$, for all $\sigma \subset I$, and for all $f \in \mathcal{H}$, we have

$$
\left(\sum_{i \in \sigma}\left\langle f, \varphi_{i}\right\rangle \overline{\left\langle f, \phi_{i}\right\rangle}\right)+\left\|\sum_{i \in \sigma^{c}}\left\langle f, \varphi_{i}\right\rangle \psi_{i}\right\|^{2}=\overline{\left(\sum_{i \in \sigma^{c}}\left\langle f, \varphi_{i}\right\rangle \overline{\left\langle f, \psi_{i}\right\rangle}\right)}+\left\|\sum_{i \in \sigma}\langle f, \varphi\rangle \phi_{i}\right\|^{2} .
$$

Proof For $\sigma \subset I$ and $f \in \mathcal{H}$, we define the operator $E_{\sigma}$ and $E_{\sigma^{c}}$ as in Theorem 9. Therefore, we have $E_{\sigma}+E_{\sigma^{c}}=I_{\mathcal{H}}$. By Lemma 1, we have

$$
\begin{aligned}
\left(\sum_{i \in \sigma}\left\langle f, \varphi_{i}\right\rangle \overline{\left\langle f, \phi_{i}\right\rangle}\right)+\left\|\sum_{i \in \sigma^{c}}\left\langle f, \varphi_{i}\right\rangle \psi_{i}\right\|^{2} & =\left\langle E_{\sigma} f, f\right\rangle+\left\langle E_{\sigma^{c}}^{*} E_{\sigma c} f, f\right\rangle \\
& =\left\langle E_{\sigma c}^{*} f, f\right\rangle+\left\langle E_{\sigma}^{*} E_{\sigma} f, f\right\rangle \\
& =\overline{\left\langle E_{\sigma^{c}}^{*} f, f\right\rangle}+\left\|E_{\sigma} f\right\|^{2} \\
& =\overline{\left(\sum_{i \in \sigma^{c}}\left\langle f, \varphi_{i}\right\rangle \overline{\left\langle f, \psi_{i}\right\rangle}\right)}+\left\|\sum_{i \in \sigma}\langle f, \varphi\rangle \phi_{i}\right\|^{2} .
\end{aligned}
$$

Hence, (2.20) holds. The proof is completed.

Theorem 12 Suppose that two frames $\left\{\phi_{i}\right\}_{i \in I}$ and $\left\{\psi_{i}\right\}_{i \in I}$ for a Hilbert space $\mathcal{H}$ are woven and $\left\{\varphi_{i}\right\}_{i \in I}$ is an alternate dual frame of the weaving frame $\left\{\phi_{i}\right\}_{i \in \sigma} \cup\left\{\phi_{i}\right\}_{i \in \sigma^{c}}$. Then, for every bounded sequence $\left\{a_{i}\right\}_{i \in I}$ and every $f \in \mathcal{H}$, we have

$$
\begin{aligned}
& \left(\sum_{i \in \sigma} a_{i}\left\langle f, \varphi_{i}\right\rangle \overline{\left\langle f, \phi_{i}\right\rangle}\right)+\left(\sum_{i \in \sigma^{c}} a_{i}\left\langle f, \varphi_{i}\right\rangle \overline{\left\langle f, \psi_{i}\right\rangle}\right) \\
& \quad+\left\|\sum_{i \in \sigma^{c}}\left(1-a_{i}\right)\left\langle f, \varphi_{i}\right\rangle \psi_{i}+\sum_{i \in \sigma}\left(1-a_{i}\right)\left\langle f, \varphi_{i}\right\rangle \phi_{i}\right\|^{2} \\
& =\left\|\sum_{i \in \sigma} a_{i}\left\langle f, \varphi_{i}\right\rangle \phi_{i}+\sum_{i \in \sigma^{c}} a_{i}\left\langle f, \varphi_{i}\right\rangle \psi_{i}\right\|^{2} \\
& \quad+\overline{\left(\sum_{i \in \sigma}\left(1-a_{i}\right)\left\langle f, \varphi_{i}\right\rangle \overline{\left\langle f, \phi_{i}\right\rangle}\right)}+\overline{\left(\sum_{i \in \sigma^{c}}\left(1-a_{i}\right)\left\langle f, \varphi_{i}\right\rangle \overline{\left\langle f, \psi_{i}\right\rangle}\right)} .
\end{aligned}
$$

Proof For all $\sigma \subset I$ and $f \in \mathcal{H}$, we define the operators

$$
E_{\sigma} f=\sum_{i \in \sigma} a_{i}\left\langle f, \varphi_{i}\right\rangle \phi_{i}, \quad E_{\sigma} f f=\sum_{i \in \sigma^{c}} a_{i}\left\langle f, \varphi_{i}\right\rangle \psi_{i}
$$

and

$$
F_{\sigma} f=\sum_{i \in \sigma}\left(1-a_{i}\right)\left\langle f, \varphi_{i}\right\rangle \phi_{i}, \quad F_{\sigma c} f=\sum_{i \in \sigma}\left(1-a_{i}\right)\left\langle f, \varphi_{i}\right\rangle \psi_{i}
$$


Note that these series converge unconditionally. We also have $E_{\sigma}, E_{\sigma^{c}}, F_{\sigma}, F_{\sigma^{c}} \in L(\mathcal{H})$ and $E_{\sigma}+E_{\sigma^{c}}+F_{\sigma}+F_{\sigma^{c}}=I_{\mathcal{H}}$. Applying Lemma 1 to the operators $P=E_{\sigma}+E_{\sigma^{c}}$ and $Q=F_{\sigma}+F_{\sigma^{c}}$, and for every $f \in \mathcal{H}$, we have

$$
\begin{aligned}
\left(\sum_{i \in \sigma} a_{i}\left\langle f, \varphi_{i}\right\rangle \overline{\left\langle f, \phi_{i}\right\rangle}\right)+\left(\sum_{i \in \sigma^{c}} a_{i}\left\langle f, \varphi_{i}\right\rangle \overline{\left\langle f, \psi_{i}\right\rangle}\right) & \\
& +\left\|\sum_{i \in \sigma^{c}}\left(1-a_{i}\right)\left\langle f, \varphi_{i}\right\rangle \psi_{i}+\sum_{i \in \sigma}\left(1-a_{i}\right)\left\langle f, \varphi_{i}\right\rangle \phi_{i}\right\|^{2} \\
= & \left\langle E_{\sigma} f, f\right\rangle+\left\langle E_{\sigma} c f, f\right\rangle+\left\langle\left(F_{\sigma}+F_{\sigma^{c}}\right)^{*}\left(F_{\sigma}+F_{\sigma^{c}}\right) f, f\right\rangle \\
= & \left\langle\left(E_{\sigma}+E_{\sigma^{c}}\right) f, f\right\rangle+\left\langle\left(F_{\sigma}+F_{\sigma^{c}}\right)^{*}\left(F_{\sigma}+F_{\sigma^{c}}\right) f, f\right\rangle \\
= & \left\langle\left(F_{\sigma}+F_{\sigma^{c}}\right)^{*} f, f\right\rangle+\left\langle\left(E_{\sigma}+E_{\sigma^{c}}\right)^{*}\left(E_{\sigma}+E_{\sigma^{c}}\right) f, f\right\rangle \\
= & \overline{\left\langle\left(F_{\sigma}+F_{\sigma^{c}}\right) f, f\right\rangle}+\left\|\left(E_{\sigma}+E_{\sigma^{c}}\right) f\right\|^{2} \\
= & \|\left(E_{\sigma}+E_{\left.\sigma^{c}\right) f} \|^{2}+\overline{\left\langle F_{\sigma} f, f\right\rangle}+\overline{\left\langle F_{\sigma} c f, f\right\rangle}\right. \\
= & \left\|\sum_{i \in \sigma} a_{i}\left\langle f, \varphi_{i}\right\rangle \phi_{i}+\sum_{i \in \sigma^{c}} a_{i}\left\langle f, \varphi_{i}\right\rangle \psi_{i}\right\|^{2} \\
& +\overline{\left(\sum_{i \in \sigma}\left(1-a_{i}\right)\left\langle f, \varphi_{i}\right\rangle \overline{\left\langle f, \phi_{i}\right\rangle}\right)}+\overline{\left(\sum_{i \in \sigma^{c}}\left(1-a_{i}\right)\left\langle f, \varphi_{i}\right\rangle \overline{\left\langle f, \psi_{i}\right\rangle}\right)} .
\end{aligned}
$$

Hence, the relation holds.

Observe that if we consider $\sigma \subset I$ and

$$
a_{i}= \begin{cases}0 & \text { if } i \in \sigma, \\ 1 & \text { if } i \in \sigma^{c}\end{cases}
$$

then Theorem 11 follows from Theorem 12.

Remark 13 If we take $\phi_{i}=\psi_{i}$ for all $i \in I$ in Theorems 11 and 12, we can obtain Theorems 4 and 2.3 of [23].

Theorem 14 Suppose that two frames $\left\{\phi_{i}\right\}_{i \in I}$ and $\left\{\psi_{i}\right\}_{i \in I}$ for a Hilbert space $\mathcal{H}$ are woven, then, for any $\lambda \in \mathcal{R}, \sigma \subset I$, and $f \in \mathcal{H}$, we have

$$
\begin{aligned}
0 & \leq \sum_{i \in \sigma}\left|\left\langle f, \phi_{i}\right\rangle\right|^{2}-\sum_{i \in \sigma}\left|\left\langle S_{\mathcal{W}}^{\sigma} f, S_{\mathcal{W}}^{-1} \phi_{i}\right\rangle\right|^{2}-\sum_{i \in \sigma^{c}}\left|\left\langle S_{\mathcal{W}}^{\sigma} f, S_{\mathcal{W}}^{-1} \psi_{i}\right\rangle\right|^{2} \\
& \leq \frac{\lambda^{2}}{4} \sum_{i \in \sigma^{c}}\left|\left\langle f, \psi_{i}\right\rangle\right|^{2}+\left(1-\frac{\lambda}{2}\right)^{2} \sum_{i \in \sigma}\left|\left\langle f, \phi_{i}\right\rangle\right|^{2} .
\end{aligned}
$$

Proof Considering positive operators $P=S_{\mathcal{W}}^{-1 / 2} S_{\mathcal{W}}^{\sigma} S_{\mathcal{W}}^{-1 / 2}$ and $Q=S_{\mathcal{W}}^{-1 / 2} S_{\mathcal{W}}^{\sigma c} S_{\mathcal{W}}^{-1 / 2}$, then $P+Q=$ $I_{\mathcal{H}}$, and

$$
P Q=P\left(I_{\mathcal{H}}-P\right)=P-P^{2}=\left(I_{\mathcal{H}}-P\right) P=Q P .
$$


Then

$$
0 \leq P Q=P\left(I_{\mathcal{H}}-P\right)=P-P^{2}=S_{\mathcal{W}}^{-1 / 2}\left(S_{\mathcal{W}}^{\sigma}-S_{\mathcal{W}}^{\sigma} S_{\mathcal{W}}^{-1} S_{\mathcal{W}}^{\sigma}\right) S_{\mathcal{W}}^{-1 / 2}
$$

from which follows $S_{\mathcal{W}}^{\sigma}-S_{\mathcal{W}}^{\sigma} S_{\mathcal{W}}^{-1} S_{\mathcal{W}}^{\sigma} \geq 0$. Then, for all $f \in \mathcal{H}$, we have

$$
\begin{aligned}
& \sum_{i \in \sigma}\left|\left\langle f, \phi_{i}\right\rangle\right|^{2}-\sum_{i \in \sigma}\left|\left\langle S_{\mathcal{W}}^{\sigma} f, S_{\mathcal{W}}^{-1} \phi_{i}\right\rangle\right|^{2}-\sum_{i \in \sigma^{c}}\left|\left\langle S_{\mathcal{W}}^{\sigma} f, S_{\mathcal{W}}^{-1} \psi_{i}\right\rangle\right|^{2} \\
& =\left\langle S_{\mathcal{W}}^{\sigma} f, f\right\rangle-\left\langle S_{\mathcal{W}}^{-1} S_{\mathcal{W}}^{\sigma} f, S_{\mathcal{W}}^{\sigma} f\right\rangle \\
& =\left\langle\left(S_{\mathcal{W}}^{\sigma}-S_{\mathcal{W}}^{\sigma} S_{\mathcal{W}}^{-1} S_{\mathcal{W}}^{\sigma}\right) f, f\right\rangle \geq 0 .
\end{aligned}
$$

By (2.4), we have

$$
\left\langle S_{\mathcal{W}}^{-1} S_{\mathcal{W}}^{\sigma} f, S_{\mathcal{W}}^{\sigma} f\right\rangle-\left\langle S_{\mathcal{W}}^{\sigma} f, f\right\rangle \geq \lambda\left\langle S_{\mathcal{W}}^{\sigma} f, f\right\rangle-\frac{\lambda^{2}}{4}\left\langle S_{\mathcal{W}} f, f\right\rangle-\left\langle S_{\mathcal{W}}^{\sigma} f, f\right\rangle
$$

and then

$$
\begin{aligned}
& \sum_{i \in \sigma}\left|\left\langle f, \phi_{i}\right\rangle\right|^{2}-\sum_{i \in \sigma}\left|\left\langle S_{\mathcal{W}}^{\sigma} f, S_{\mathcal{W}}^{-1} \phi_{i}\right\rangle\right|^{2}-\sum_{i \in \sigma^{c}}\left|\left\langle S_{\mathcal{W}}^{\sigma} f, S_{\mathcal{W}}^{-1} \psi_{i}\right\rangle\right|^{2} \\
& =\left\langle S_{\mathcal{W}}^{\sigma} f, f\right\rangle-\left\langle S_{\mathcal{W}}^{-1} S_{\mathcal{W}}^{\sigma} f, S_{\mathcal{W}}^{\sigma} f\right\rangle \\
& \leq\left\langle S_{\mathcal{W}}^{\sigma} f, f\right\rangle-\lambda\left\langle S_{\mathcal{W}}^{\sigma} f, f\right\rangle+\frac{\lambda^{2}}{4}\left\langle S_{\mathcal{W}} f, f\right\rangle \\
& =(1-\lambda)\left\langle S_{\mathcal{W}}^{\sigma} f, f\right\rangle+\frac{\lambda^{2}}{4}\left\langle S_{\mathcal{W}} f, f\right\rangle \\
& =(1-\lambda)\left\langle\left(S_{\mathcal{W}}-S_{\mathcal{W}}^{\sigma^{c}}\right) f, f\right\rangle+\frac{\lambda^{2}}{4}\left\langle S_{\mathcal{W}} f, f\right\rangle \\
& =(\lambda-1)\left\langle S_{\mathcal{W}}^{\sigma^{c}} f, f\right\rangle+\left(1-\frac{\lambda}{2}\right)^{2}\left\langle S_{\mathcal{W}} f, f\right\rangle \\
& =\frac{\lambda^{2}}{4}\left\langle S_{\mathcal{W}}^{\sigma^{c}} f, f\right\rangle+\left(1-\frac{\lambda}{2}\right)^{2}\left\langle S_{\mathcal{W}}^{\sigma} f, f\right\rangle \\
& =\frac{\lambda^{2}}{4} \sum_{i \in \sigma^{c}}\left|\left\langle f, \psi_{i}\right\rangle\right|^{2}+\left(1-\frac{\lambda}{2}\right)^{2} \sum_{i \in \sigma}\left|\left\langle f, \phi_{i}\right\rangle\right|^{2} .
\end{aligned}
$$

Theorem 15 Suppose that two frames $\left\{\phi_{i}\right\}_{i \in I}$ and $\left\{\psi_{i}\right\}_{i \in I}$ for a Hilbert space $\mathcal{H}$ are woven, then, for any $\lambda \in \mathcal{R}, \sigma \subset I$, and $f \in \mathcal{H}$, we have

$$
\begin{aligned}
(2 \lambda & \left.-\frac{\lambda^{2}}{2}-1\right) \sum_{i \in \sigma}\left|\left\langle f, \phi_{i}\right\rangle\right|^{2}+\left(1-\frac{\lambda^{2}}{2}\right) \sum_{i \in \sigma^{c}}\left|\left\langle f, \psi_{i}\right\rangle\right|^{2} \\
\leq & \sum_{i \in \sigma}\left|\left\langle S_{\mathcal{W}}^{\sigma} f, S_{\mathcal{W}}^{-1} \phi_{i}\right\rangle\right|^{2}+\sum_{i \in \sigma^{c}}\left|\left\langle S_{\mathcal{W}}^{\sigma} f, S_{\mathcal{W}}^{-1} \psi_{i}\right\rangle\right|^{2} \\
& +\sum_{i \in \sigma}\left|\left\langle S_{\mathcal{W}}^{\sigma^{c}} f, S_{\mathcal{W}}^{-1} \phi_{i}\right\rangle\right|^{2}+\sum_{i \in \sigma^{c}}\left|\left\langle S_{\mathcal{W}}^{\sigma_{\mathcal{W}}^{c}} f, S_{\mathcal{W}}^{-1} \psi_{i}\right\rangle\right|^{2} \\
\leq & \sum_{i \in \sigma}\left|\left\langle f, \phi_{i}\right\rangle\right|^{2}+\sum_{i \in \sigma^{c}}\left|\left\langle f, \psi_{i}\right\rangle\right|^{2} .
\end{aligned}
$$


Proof By (2.5), we have

$$
\begin{aligned}
& \left\langle S_{\mathcal{W}}^{-1} S_{\mathcal{W}}^{\sigma} f, S_{\mathcal{W}}^{\sigma} f\right\rangle \geq\left(\lambda-\frac{\lambda^{2}}{4}\right)\left\langle S_{\mathcal{W}}^{\sigma} f, f\right\rangle-\frac{\lambda^{2}}{4}\left\langle S_{\mathcal{W}}^{\sigma^{c}} f, f\right\rangle, \\
& \left\langle S_{\mathcal{W}}^{-1} S_{\mathcal{W}}^{\left.\sigma^{c} f, S_{\mathcal{W}}^{\sigma^{c}} f\right\rangle} \geq\left(\lambda-\frac{\lambda^{2}}{4}-1\right)\left\langle S_{\mathcal{W}}^{\sigma} f, f\right\rangle+\left(1-\frac{\lambda^{2}}{4}\right)\left\langle S_{\mathcal{W}}^{\sigma^{c}} f, f\right\rangle .\right.
\end{aligned}
$$

From (2.21) and (2.21), we obtain

$$
\begin{aligned}
& \sum_{i \in \sigma}\left|\left\langle S_{\mathcal{W}}^{\sigma} f, S_{\mathcal{W}}^{-1} \phi_{i}\right\rangle\right|^{2}+\sum_{i \in \sigma^{c}}\left|\left\langle S_{\mathcal{W}}^{\sigma} f, S_{\mathcal{W}}^{-1} \psi_{i}\right\rangle\right|^{2}+\sum_{i \in \sigma}\left|\left\langle S_{\mathcal{W}}^{\sigma^{c}} f, S_{\mathcal{W}}^{-1} \phi_{i}\right\rangle\right|^{2}+\sum_{i \in \sigma^{c}}\left|\left\langle S_{\mathcal{W}}^{\sigma^{c}} f, S_{\mathcal{W}}^{-1} \psi_{i}\right\rangle\right|^{2} \\
& \quad=\left\langle S_{\mathcal{W}}^{-1} S_{\mathcal{W}}^{\sigma} f, S_{\mathcal{W}}^{\sigma} f\right\rangle+\left\langle S_{\mathcal{W}}^{-1} S_{\mathcal{W}}^{\sigma^{c}} f, S_{\mathcal{W}}^{\sigma^{c}} f\right\rangle \\
& \quad \geq\left(2 \lambda-\frac{\lambda^{2}}{2}-1\right)\left\langle S_{\mathcal{W}}^{\sigma} f, f\right\rangle+\left(1-\frac{\lambda^{2}}{2}\right)\left\langle S_{\mathcal{W}}^{\sigma^{c}} f, f\right\rangle \\
& \quad=\left(2 \lambda-\frac{\lambda^{2}}{2}-1\right) \sum_{i \in \sigma}\left|\left\langle f, \phi_{i}\right\rangle\right|^{2}+\left(1-\frac{\lambda^{2}}{2}\right) \sum_{i \in \sigma^{c}}\left|\left\langle f, \psi_{i}\right\rangle\right|^{2} .
\end{aligned}
$$

Next, we prove the last part. Let $P=S_{\mathcal{W}}^{-1 / 2} S_{\mathcal{W}}^{\sigma} S_{\mathcal{W}}^{-1 / 2}, Q=S_{\mathcal{W}}^{-1 / 2} S_{\mathcal{W}}^{\sigma^{c}} S_{\mathcal{W}}^{-1 / 2}$. Since $P Q=Q P$, we have

$$
P-P^{2}=P\left(I_{\mathcal{H}}-P\right)=P Q \geq 0,
$$

and then, for all $f \in \mathcal{H},\|P f\|^{2} \leq\langle P f, f\rangle$. Similarly, $\|Q f\|^{2} \leq\langle Q f, f\rangle$. Hence,

$$
\begin{aligned}
\sum_{i \in \sigma} & \left|\left\langle S_{\mathcal{W}}^{\sigma} f, S_{\mathcal{W}}^{-1} \phi_{i}\right\rangle\right|^{2}+\sum_{i \in \sigma^{c}}\left|\left\langle S_{\mathcal{W}}^{\sigma} f, S_{\mathcal{W}}^{-1} \psi_{i}\right\rangle\right|^{2}+\sum_{i \in \sigma}\left|\left\langle S_{\mathcal{W}}^{\sigma^{c}} f, S_{\mathcal{W}}^{-1} \phi_{i}\right\rangle\right|^{2}+\sum_{i \in \sigma^{c}}\left|\left\langle S_{\mathcal{W}}^{\sigma^{c}} f, S_{\mathcal{W}}^{-1} \psi_{i}\right\rangle\right|^{2} \\
= & \left\langle S_{\mathcal{W}}^{-1} S_{\mathcal{W}}^{\sigma} f, S_{\mathcal{W}}^{\sigma} f\right\rangle+\left\langle S_{\mathcal{W}}^{-1} S_{\mathcal{W}}^{\sigma^{c}} f, S_{\mathcal{W}}^{\sigma^{c}} f\right\rangle \\
& =\left\langle S_{\mathcal{W}}^{-1 / 2} S_{\mathcal{W}}^{\sigma} f, S_{\mathcal{W}}^{-1 / 2} S_{\mathcal{W}}^{\sigma} f\right\rangle+\left\langle S_{\mathcal{W}}^{-1 / 2} S_{\mathcal{W}}^{\sigma^{c}} f, S_{\mathcal{W}}^{-1 / 2} S_{\mathcal{W}}^{\sigma^{c}} f\right\rangle \\
& =\left\langle S_{\mathcal{W}}^{-1 / 2} S_{\mathcal{W}}^{\sigma} S_{\mathcal{W}}^{-1 / 2} S_{\mathcal{W}}^{1 / 2} f, S_{\mathcal{W}}^{-1 / 2} S_{\mathcal{W}}^{\sigma} S_{\mathcal{W}}^{-1 / 2} S_{\mathcal{W}}^{1 / 2} f\right\rangle+\left\langle S_{\mathcal{W}}^{-1 / 2} S_{\mathcal{W}}^{\sigma c} S_{\mathcal{W}}^{-1 / 2} S_{\mathcal{W}}^{1 / 2} f, S_{\mathcal{W}}^{-1 / 2} S_{\mathcal{W}}^{\sigma c} S_{\mathcal{W}}^{-1 / 2} S_{\mathcal{W}}^{1 / 2} f\right\rangle \\
& \leq\left\langle S_{\mathcal{W}}^{-1 / 2} S_{\mathcal{W}}^{\sigma} S_{\mathcal{W}}^{-1 / 2} S_{\mathcal{W}}^{1 / 2} f, S_{\mathcal{W}}^{1 / 2} f\right\rangle+\left\langle S_{\mathcal{W}}^{-1 / 2} S_{\mathcal{W}}^{\sigma c} S_{\mathcal{W}}^{-1 / 2} S_{\mathcal{W}}^{1 / 2} f, S_{\mathcal{W}}^{1 / 2} f\right\rangle \\
& =\left\langle S_{\mathcal{W}}^{\sigma} f, f\right\rangle+\left\langle S_{\mathcal{W}}^{\sigma^{c}} f, f\right\rangle \\
& =\sum_{i \in \sigma}\left|\left\langle f, \phi_{i}\right\rangle\right|^{2}+\sum_{i \in \sigma}\left|\left\langle f, \psi_{i}\right\rangle\right|^{2} .
\end{aligned}
$$

By Theorems 14 and 15, we immediately obtain the following results.

Corollary 1 Suppose that two frames $\left\{\phi_{i}\right\}_{i \in I}$ and $\left\{\psi_{i}\right\}_{i \in I}$ for a Hilbert space $\mathcal{H}$ are A-woven, then, for any $\lambda \in \mathcal{R}, \sigma \subset I$, and $f \in \mathcal{H}$, we have

$$
0 \leq A \sum_{i \in \sigma}\left|\left\langle f, \phi_{i}\right\rangle\right|^{2}-\left\|\sum_{i \in \sigma}\left\langle f, \phi_{i}\right\rangle \phi_{i}\right\|^{2} \leq \frac{A \lambda^{2}}{4} \sum_{i \in \sigma^{c}}\left|\left\langle f, \psi_{i}\right\rangle\right|^{2}+\left(1-\frac{\lambda}{2}\right)^{2} A \sum_{i \in \sigma}\left|\left\langle f, \phi_{i}\right\rangle\right|^{2}
$$

and

$$
\left(2 \lambda-\frac{\lambda^{2}}{2}-1\right) A \sum_{i \in \sigma}\left|\left\langle f, \phi_{i}\right\rangle\right|^{2}+\left(1-\frac{\lambda^{2}}{2}\right) A \sum_{i \in \sigma^{c}}\left|\left\langle f, \psi_{i}\right\rangle\right|^{2}
$$




$$
\leq\left\|\sum_{i \in \sigma}\left\langle f, \phi_{i}\right\rangle \phi_{i}\right\|^{2}+\left\|\sum_{i \in \sigma^{c}}\left\langle f, \psi_{i}\right\rangle \psi_{i}\right\|^{2} \leq A\|f\|^{2} .
$$

Proof Since $\left\{\phi_{i}\right\}_{i \in I}$ and $\left\{\psi_{i}\right\}_{i \in I}$ are $A$-woven, we have $S_{\mathcal{W}}^{-1}=\frac{1}{A} I_{\mathcal{H}}$, and then the results hold by Theorems 14 and 15 .

Remark 16 If we take $\lambda=1$ and $\phi_{i}=\psi_{i}$ for all $i \in I$ in Theorems 14 and 15, we obtain the similar inequalities in Theorems 5 and 6 of [14].

\section{Acknowledgements}

The authors thank the reviewers for their valuable comments.

Funding

The research is supported by the Natural Science Foundation of Anhui Province (1908085MF175).

Availability of data and materials

Not applicable.

\section{Declarations}

Competing interests

The authors declare that they have no competing interests.

\section{Authors' contributions}

YX contributed significantly to analysis and manuscript preparation; JL helped perform the analysis with constructive discussions. All authors read and approved the final manuscript.

\section{Publisher's Note}

Springer Nature remains neutral with regard to jurisdictional claims in published maps and institutional affiliations.

Received: 7 July 2021 Accepted: 9 November 2021 Published online: 26 November 2021

\section{References}

1. Balan, R., Casazza, P., Edidin, D.: On signal reconstruction without phase. Appl. Comput. Harmon. Anal. 20(3), 345-356 (2006)

2. Balan, R., Casazza, P., Edidin, D., Kutyniok, G.: Decompositions of frames and a new frame identity. Proc. SPIE 5914, $1-10(2005)$

3. Balan, R., Casazza, P., Edidin, D., Kutyniok, G.: A new identity for Parseval frames. Proc. Am. Math. Soc. 135(4), 1007-1015 (2007)

4. Bemrose, T., Casazza, P.G., Gröchenig, K., Lammers, M.C., Lynch, R.G.: Weaving frames (2015). arXiv preprint arXiv:1503.03947

5. Casazza, P.G., Freeman, D., Lynch, R.G.: Weaving Schauder frames. J. Approx. Theory 211, $42-60$ (2016)

6. Daubechies, I., Grossmann, A., Meyer, Y.: Painless nonorthogonal expansions. J. Math. Phys. 27(5), 1271-1283 (1986)

7. Donoho, D., Candes, E.: Continuous curvelet transform: II. Discretization and frames. Appl. Comput. Harmon. Anal. 19(2), 198-222 (2005)

8. Duffin, R.J., Schaeffer, A.C.: A class of nonharmonic Fourier series. Trans. Am. Math. Soc. 72(2), 341-366 (1952)

9. Eldar, Y.C., Forney, G.D.: Optimal tight frames and quantum measurement. IEEE Trans. Inf. Theory 48(3), 599-610 (2002)

10. Găvruţa, P.: On some identities and inequalities for frames in Hilbert spaces. J. Math. Anal. Appl. 321(1), 469-478 (2006)

11. Han, D., Larson, D.R.: Frames, Bases and Group Representations, vol. 697. Am. Math. Soc., Providence (2000)

12. Khosravi, A., Sohrabi Banyarani, J.: Weaving g-frames and weaving fusion frames. Bull. Malays. Math. Sci. Soc. 42 , 3111-3129 (2019)

13. Kovacevic, J., Dragotti, P.L., Goyal, V.K.: Filter bank frame expansions with erasures. IEEE Trans. Inf. Theory 48(6), 1439-1450 (2002)

14. Li, D., Leng, J.: On some new inequalities for fusion frames in Hilbert spaces. Math. Inequal. Appl. 20(3), 889-900 (2017)

15. Li, D., Leng, J.: On some new inequalities for continuous fusion frames in Hilbert spaces. Mediterr. J. Math. 15(4), 173 (2018)

16. Li, D., Leng, J., Huang, T., Gao, Q.: Frame expansions with probabilistic erasures. Digit. Signal Process. 72, 75-82 (2018)

17. Li, J.-Z., Zhu, Y.-C.: Some equalities and inequalities for g-Bessel sequences in Hilbert spaces. Appl. Math. Lett. 25(11), 1601-1607 (2012)

18. Poria, A.: Some identities and inequalities for Hilbert-Schmidt frames. Mediterr. J. Math. 14(2), 59 (2017)

19. Sun, Q., Tang, W.-S.: Nonlinear frames and sparse reconstructions in Banach spaces. J. Fourier Anal. Appl. 23(5), 1118-1152 (2017)

20. Vashisht, L.K., et al.: Weaving properties of generalized continuous frames generated by an iterated function system. J. Geom. Phys. 110, 282-295 (2016) 
21. Xiao, X., Zhu, Y., Ding, M.: Erasures and equalities for fusion frames in Hillbert spaces. Bull. Malays. Math. Sci. Soc. 38(3), 1035-1045 (2015)

22. Zhao, P., Zhao, C., Casazza, P.G.: Perturbation of regular sampling in shift-invariant spaces for frames. IEEE Trans. Inf. Theory 52(10), 4643-4648 (2006)

23. Zhu, X., Wu, G.: A note on some equalities for frames in Hilbert spaces. Appl. Math. Lett. 23(7), 788-790 (2010)

Submit your manuscript to a SpringerOpen ${ }^{\circ}$ journal and benefit from:

- Convenient online submission

$\checkmark$ Rigorous peer review

- Open access: articles freely available online

- High visibility within the field

- Retaining the copyright to your article

Submit your next manuscript at $\gg$ springeropen.com 